\title{
Análisis de pastillas como prevención selectiva
}

\author{
Gregor Burkhart*, Harald Kriener** \\ ${ }^{*}$ OEDT $^{1},{ }^{*}$ ISP Viena \\ Enviar correspondencia: European Monitoring Centre for Drugs and Drug Addiction. da Cruz de Sta. Apolónia 23-25. \\ P-1149-045 Lisboa - Portugal. Telef. +351 21811 3022. Gregor.Burkhart@emcdda.eu.int. Http://www.emcdda.eu.int
}

\section{Resumen}

En los últimos años, ha habido una creciente preocupación en la Unión Europea por el contenido de las pastillas que se venden bajo el nombre de éxtasis, debido a que éstas muchas veces podrían estar compuestas por otro tipo de sustancias.

Sin embargo, es muy difícil tomar las medidas de prevención puesto que los consumidores de éxtasis constituyen un grupo diana al que es difícil llegar para informarles sobre los riesgos de salud ya que estas personas no acceden a los sistemas tradicionales de prevención. El análisis de pastillas originalmente surgió como respuesta específica a la adulteración de las pastillas pero ahora se revela también como una estrategia innovadora para alcanzar grupos de riesgo específicos. Si bien algunos países cuentan con información comparable sobre el modo de operación y los objetivos de los proyectos de análisis de pastillas, el debate sobre este tema sigue siendo candente ideológico. En este artículo se revisan los argumentos más frecuentes que giran en torno a estas intervenciones. La semi-legalidad de muchos de estos proyectos es, a su vez, causa y efecto de la escasez de evaluaciones más rigurosas en este campo. No obstante, la información disponible en este momento permite definir ciertos criterios de calidad (estrategia, formación, equipamiento) y de investigación.

Palabras claves: Análisis de pastillas, Éxtasis, prevención secundaria, drogas sintéticas, reducción del riesgo

\section{Summary}

Concern in the European Union has increased during the past years about the content of pills sold as ecstasy.

However, within this context, the challenge of taking the necessary prevention measures is great in particular to approach and inform hard-to-reach target groups such as ecstasy consumers about health risks, as these groups do not access the traditional drug care systems. On-site pill testing originally emerged as a concrete response to the adulteration of pills commonly sold as ecstasy but is revealing now as an innovative means to approach specific risk groups. Although some countries nowadays offer comparable information regarding modus operandi, contents and aims of on-site pill testing projects, discussions about this issue are still very vivid and, sometimes, ideological. The present article's aim is to provide an objective overview of goals, methods, results and evaluation efforts of pill-testing projects. This overview gathers and discusses the most common opinions about these projects. The pseudo-legality of most projects is both the cause and consequence of the lack of rigorous evaluations in this area. Nevertheless, the information available nowadays in Europe opens the possibility of defining research and quality criteria (strategy, training, equipment) for pill testing projects.

Key Words: Pill-testing, drug-checking, ecstasy, secondary prevention, synthetic drugs, harm reduction.

(1) La tarea del Observatorio Europeo de las Drogas y Toxicomanías (OEDT) es informar activamente al público, a los políticos y a los profesionales en general sobre las nuevas tendencias y prácticas que emergen en los estados miembro o en algunas regiones, a fin de propiciar el debate y el intercambio de ideas sobre posibles estrategias innovadoras. El presente texto no constituye ninguna declaración formal o recomendación por parte del Observatorio Europeo ni a favor ni en contra de la implementación de dichas medidas, a menos que haya evidencia suficiente sobre su beneficio o efectividad. Por lo tanto, el Observatorio Europeo rechaza expresamente cualquier acusación de promoción o apoyo específico a cualquiera de las políticas, estrategias o filosofías que aquí se presentan. 
e denomina "análisis de pastillas in situ" al conjunto heterogéneo de actividades que se realiza para analizar las sustancias contenidas dentro de las pastillas que se venden en los lugares de ocio, tal es el caso del éxtasis. El objetivo es brindar a los potenciales consumidores los resultados dentro de la misma fiesta y, por lo general, se hace de un modo inmediato.

Esta iniciativa surgió en el marco de una política de reducción de daños y se basa en la hipótesis de que en las discotecas europeas suele venderse como éxtasis pastillas que no contienen la sustancia (MDMA), o la contienen sólo en una dosis reducida (OFDT 1999) y que posiblemente estén compuestas por anfetaminas o, incluso, por sustancias más peligrosas.

\section{A. OBJETIVOS DE LOS ACTUALES PRO- YECTOS DE ANÁLISIS DE PASTILLAS}

El análisis de pastillas puede tener diferentes objetivos. En un sentido general, se lo usa para prevenir acerca de sustancias dañinas e inesperadas, tanto in situ o a través de Internet, puesto que representa un buen modo de contactar potenciales consumidores de sustancias ilícitas para brindarles información y apoyo. Por otra parte, el análisis de pastillas proporciona una información precisa acerca de la situación en el mercado negro actual y además permite detectar, hacer un seguimiento y controlar las nuevas tendencias de consumo, los patrones de uso locales y ofrece, además, una amplia información demográfica sobre consumidores de sustancias ilícitas y otras personas en riesgo.

El objetivo de DIMS, en Holanda, (http:// www.trimbos.nl) es prevenir daños de salud por sobredosis o toxicidad de las substancias y crear una red de información nacional. Por su lado, el proyecto ChEck-It, de Austria, (http://www.checkyourdrugs.at/) proporciona resultados inmediatos de los análisis y utiliza esta ocasión para entrar en contacto y discusión con quienes traen sus pastillas para analizar. En este caso, se emplean los análisis con múltiples propósitos que van más allá de conocer el contenido de las pastillas, como por ejemplo para la investigación sobre pautas, razones de consumo y, sobre todo, para charlas de concienciación y prevención.

\section{Reducción de Daños y Riesgos}

Cuando se habla de reducción de daños "se hace referencia a las políticas y programas enfocados a la reducción de daños por uso de alcohol y otras drogas, tanto desde el punto de vista individual como dentro de la comunidad."2

La reducción de riesgos "describe estrategias o programas que apuntan a disminuir el daño que producen el alcohol y otras drogas. Las estrategias de reducción de riesgos tienen algunas ventajas prácticas puesto que generalmente es más fácil medir con objetividad los comportamientos riesgosos que los daños, especialmente si se trata de daños que se evidencian con una frecuencia baja."

En la actualidad, se cuenta con algunas acciones de reducción de daños que responden a las nuevas drogas sintéticas, tales como repartir preservativos o proporcionar agua potable para controlar la temperatura corporal y evitar los golpes de calor. A diferencia de otros proyectos los proyectos de análisis de pastillas tienen la capacidad de hacerle frente en las fiestas a algunos potenciales daños ya que informan in situ a los consumidores acerca de las pastillas peligrosas o con contenidos inesperados, y expanden esta información mediante revistas, afiches o por Internet.

\footnotetext{
(2) UNODCCP (2000), Demand Reduction. A Glossary of Terms. New York: United Nations Publications, p.31

(3) Loc.cit, p. 64. Dado que estas definiciones no difieren sustancialmente entre sí, y que algunas organizaciones prefieren el término reducción del daño que reducción del riesgo, ambos términos serán utilizados como sinónimos en este artículo. Por ejemplo en la reunion de Viena sobre Pill-testing algunos expertos especificaron que el término daño debería refererirse a situaciones objetivas mientras que reducción de riesgos se debería usar para los individuos y las decisiones que ellos toman libremente..
} 
El proyecto holandés DIMS, por ejemplo, previene acerca de pastillas compuestas con una dosis muy elevada y, al igual que el proyecto Contact Bern, previene acerca de pastiIlas que contienen atropina. En el otoño de 2000, ChEckiT! (Austria) encontró una gran cantidad de pastillas que contenían PMA/ PMMA e, inmediatamente, expandió la información por Internet y, gracias a la colaboración de los organizadores y los DJs, también en los propios locales. Esta acción hizo que 24 horas más tarde el aviso fuera publicado en las páginas web sobre prevención más importantes de Europa. ${ }^{4}$

Además de los avisos publicados sobre pastillas peligrosas o con contenidos inesperados, es muy importante tener en cuenta la dosis que contienen. Desde el punto de vista de la neurotoxicidad, varios estudios científicos han señalado que, entre otros factores, la probabilidad de daño neurotóxico en el sistema serotoninérgico aumenta de acuerdo con la cantidad de MDMA que se consume. De allí que la mayoría de los proyectos de análisis de pastillas informan a los potenciales consumidores que, en caso de consumir, no deberían exceder los 1,5-1,8 mg de MDMA por kilo de peso corporal, debido a que podría dañarse a largo plazo una región importante del cerebro. Toda esta información, que generalmente es recibida por los consumidores de éxtasis, sirve sólo si el consumidor hace analizar químicamente sus pastillas. Caso contrario, esa información carece de valor práctico. ${ }^{5}$

\section{Promoción de trabajos de prevención y mensajes para un consumo seguro}

A pesar de que una gran cantidad de habituales de fiestas raves muestra interés por conocer los efectos y peligros de las sustancias psicoactivas, captar la atención de estas personas no es tarea fácil puesto que en estos lugares se compite con muchas otras actividades muy llamativas. Por todo esto, no resulta extraño que los proyectos que sólo suministran información mediante panfletos o hablando con la gente, no siempre logren captar la atención deseada.

El análisis de pastillas constituye un instrumento muy atractivo porque trata con las sustancias, implica un equipamiento técnico $y$, sobre todo, porque a todos les interesa conocer la composición de sus pastillas. Es entonces cuando se puede aprovechar para repartir entre los habituales folletos informativos, brindar información y charlas de apoyo. En el caso de ChEckiT!, por ejemplo, se ha comprobado que cuando el proyecto se presenta en clubes o fiestas raves sólo para suministrar información, los miembros del equipo logran conversar con entre 20 y 40 personas a lo largo de 8 horas, dependiendo del local y de las personas que concurren. Sin embargo, cuando el proyecto incluye también el análisis químico de las pastillas, se logra contactar un promedio de 260 personas, mientras que "sólo" se analizan 75 pastillas. Esto demuestra que el análisis de pastillas, obviando sus beneficios específicos, constituye un buen método para impartir mensajes de consumo seguro, apoyo y realizar un trabajo de prevención en general.

No obstante, los proyectos de análisis de pastillas no pretenden ser considerados como un anzuelo para atraer a las personas. La credibilidad y la anuencia son condiciones sine qua non tanto para el análisis de las pastillas como para el apoyo psicológico. No se debe olvidar que la publicidad constituye también un efecto secundario útil e importante en estos proyectos. Tossmann y Heckmann señalan que los habituales de estas fiestas expresan que las tácticas de márketing son útiles a la hora de trabajar en prevención: "La prevención debe tomarse como un producto a presentar ante el público usando tácticas

\footnotetext{
(4) Las pastillas con PMA/PMMA produjeron más de diez muertes en los locales bailables de EEUU, Dinamarca, Noruega, Alemania y Austria Se supone que las víctimas consumieron éxtasis y murieron por causa de una falla orgánica provocada por el aumento de la temperatura corporal.

(5) Para llegar a los potenciales consumidores de sustancias ilícitas es necesario que los proyectos de análisis de pastillas, la información y el apoyo se proporcionen en aquellos lugares donde estas personas eligen pasar su tiempo de ocio, tal es el caso de las raves. Para mayor información acerca de escenarios útiles, consultar Tossmann \& Heckmann 1997, pg. 122.
} 
de márketing" (Tossmann y Heckmann 1997, pg.122).

Consejos para transmitir mensajes de consumo seguro (Forum Européen 1999):

- Los mensajes deben estar adaptados a la población receptora. Esto implica también hacer una diferenciación de sexo, puesto que los hombres y las mujeres tienen diferentes necesidades.

- Adaptar el vocabulario. Cuando se trata con jóvenes debe evitarse la terminología obscura y científica y optar por un lenguaje claro.

- Incluir en los mensajes un mínimo de información sobre reducción de daños, tratado de un modo neutro y sin connotaciones morales ni juzgamientos.

- La información debe ser breve.

- En prevención, cada tipo de droga necesita un abordaje diferente.

- La información debe estar disponible en todos los lugares de ocio que frecuentan los jóvenes, ya que esto los ayudará a estar informados antes de tomar cualquier tipo de decisión.

Los mensajes varían de sustancia a sustancia y deben reformularse de acuerdo con el grupo receptor. El quid de la cuestión es, tal como ya se lo mencionó, impartir información de un modo neutro, sin implicaciones morales ni juzgamientos y sin subestimar o alarmar al receptor.

Ejemplos de posibles mensajes de consumo seguro:

- Si a pesar de los riesgos legales y físicos que pueda acarrearte, decides consumir sustancias ilícitas, infórmate acerca de los efectos y peligros.

- El único modo de conocer la composición de una pastilla es mediante un análisis químico.

- La dosis es importante. Las mujeres logran experimentar la misma sensación que los hombres, consumiendo una dosis más pequeña.

- Cuando se mezcla sustancias, se hace imposible calcular los efectos y riesgos.
- La mayoría de las sustancias psicoactivas provocan tensiones en el organismo, por lo que no deberías consumir si tienes problemas de salud, tales como afecciones en el hígado o en los riñones.

- Los efectos que provocan las sustancias psicoactivas no obedecen sólo a la sustancia y a la cantidad que consumes. El lugar y el momento constituyen también un factor importante.

- Sé cuidadoso con tus amigos y con las personas que te rodean.

- Coméntale a tus amigos la cantidad y sustancia que has ingerido.

- Bebe suficiente líquido no alcohólico para reabastecer los fluidos de tu cuerpo.

- Descansa de tanto en tanto para permanecer calmo y relajado.

Mensajes para un sexo más seguro

- La hepatitis, el HIV y algunas otras enfermedades infecciosas pueden transmitirse sexualmente. Algunas drogas hacen que se pierda ciertas inhibiciones, por lo que debes recordar siempre usar preservativo al tener sexo.

Mensajes para un comportamiento más seguro a la hora de conducir

- Las sustancias psicoactivas podrían disminuir tu coordinación y reflejo. No conduzcas bajo el efecto de drogas.

Riesgos para la salud a largo plazo y adicciones

- Muchas sustancias ilícitas son adictivas desde el punto de vista físico y/o psicológico.

- Hay muchas sustancias que podrían provocar daño físico a largo plazo.

- El éxtasis, la metanfetamina y algunas otras drogas podrían alterar o dañar el funcionamiento del cerebro.

- La mayoría de las sustancias psicoactivas atraviesa la placenta y llega a la leche materna.

Riesgos legales

- La ley prohíbe la mayoría de las sustancias psicoactivas. Si alguien es condenado por posesión de sustancias ilícitas, corre el riesgo de pagar multas, de ir a prisión, de 
que le invaliden el registro de conducir y de que le prohíban llevar a cabo cierto tipo de trabajos.

\section{Las campañas Safer House}

El análisis de pastillas ha servido de cimiento para otros proyectos que intentan reducir los riesgos en fiestas y propiciar un ambiente más orientado hacia la salud. A modo de ejemplo, se expone el caso de la campaña holandesa Safer House que, a principios de los años '90, creó un sistema que iba mucho más allá del simple análisis de pastillas y el suministro de información. Este proyecto se aseguraba de que los organizadores de las fiestas raves se ocuparan de que el lugar estuviera bien ventilado, de que hubiera suficiente agua, amplios guardarropas, baños limpios y bebidas no alcohólicas baratas, entre otros detalles ${ }^{6}$. El personal de las compañías de seguridad y asistencia médica debía pasar por un entrenamiento específico y contar con empleadas femeninas también. Esto era un punto muy importante, especialmente para las compañías de seguridad, puesto que en general, sólo tienen en sus listas empleados masculinos. En los megaeventos, los análisis de pastillas y la diseminación de información debía realizarse de forma inmediata. El objetivo de las campañas para una fiesta rave segura, o safer house, no se reduce sólo a evitar el consumo riesgoso, sino también a crear espacios en los que se pueda disfrutar de una fiesta y, en caso de consumir drogas ilícitas, hacerlo sin correr riesgos.

\section{Información básica para la prevención}

La información que se obtiene a partir de los estudios de las drogas, el monitoreo del contenido de las pastillas, la información demográfica, las causas de consumo y las tendencias constituye una fuente esencial para reconsiderar, adaptar o ampliar los esfuerzos dentro de la prevención secunda- ria. En cuanto a la prevención primaria, la información sobre causas de consumo, tendencias e información demográfica debería utilizarse al menos para recalcular la validez de los factores de riesgo y protección específicos. Esto es muy importante pues en los últimos años ha surgido una nueva realidad en torno a los consumidores de drogas ilícitas: existen nuevas sustancias y nuevas tendencias que no pueden ser ignoradas a la hora de trabajar en prevención primaria. En general, los proyectos que surgieron en el ambiente techno, tales como Eve \& Rave, Techno Plus o Energy Control, por citar sólo algunos ejemplos, fueron piezas clave para definir las necesidades y problemática dentro de las fiestas raves, el modo de enfrentar estos problemas, la necesidad de proporcionar un espacio placentero y sano para las fiestas o festivales techno, y para formular mensajes de reducción de daños de un modo directo e inteligible. El intercambio dialéctico entre los proyectos independientes y los que están patrocinados por el Estado ayudó a recaudar mucha información, experiencias y métodos específicos. Es importante recordar que, con excepción de algunos pocos informes anecdóticos lanzados por los medios de comunicación, el público en general y los expertos en prevención todavía saben muy poco sobre métodos, objetivos y estado legal de los proyectos de análisis de pastillas.

\section{Monitoreo e Investigación}

Para recaudar información acerca del mercado de las drogas, o de las variables ${ }^{7}$ demográficas, psicológica, médicas y sociales de los habitués de fiestas raves y de otros consumidores de sustancias ilícitas, es menester establecer y mejorar los métodos de información de los proyectos de prevención, y planear los estudios científicos sobre patrones de uso y riesgos asociados. El monitoreo no implica sólo el rastreo de las sustancias, sino también la investigación de las necesidades personales, culturales, problemas y otros fac- 
tores. La recaudación efectiva de toda esta información es lo que les posibilita a aquellos que trabajan en prevención y en salud pública responder rápida y adecuadamente ante las nuevas tendencias ${ }^{8}$.

Todos los proyectos de análisis de pastillas reciben regularmente alguna información básica sobre las sustancias que se presentan para analizar y los potenciales consumidores. En general, las personas interesadas en los proyectos de análisis de pastillas se muestran bien predispuestas para participar en estudios basados en cuestionarios.

Por otra parte, estos proyectos también inspiran credibilidad ante los potenciales consumidores. Se ha comprobado que para trabajar con consumidores de sustancias ilícitas recreativos es necesario conocer perfectamente los efectos y peligros de las drogas sintéticas. Sin embargo, el único modo que existe de saber los posibles efectos que una sustancia específica, con una dosis determinada, es cuando se conoce la composición exacta de las pastillas. Caso contrario, los profesionales que diseminan la información no serán considerados como "mensajeros" fehacientes, y se tenderá a creer en todos los mitos que pululan en torno de la prevención in situ. ${ }^{9}$

\section{Requisitos previos para los sistemas de información y alerta}

Todos los sistemas de información y alerta sobre pastillas con contenidos inesperados o muy peligrosos se basan principalmente la análisis de pastillas. En la actualidad, no existe ningún otro proyecto u organización que informe sobre la situación real de las drogas y las tendencias de consumo tan rápidamente como lo hacen estos proyectos. La informa- ción suministrada por los sistemas de prevención sólo sirve cuando se le puede explicar a la gente contra qué se los advierte específicamente.

\section{Información para toda la población}

Para informar sobre los problemas relacionados con las drogas sintéticas y los proyectos de prevención a una porción más amplia de la población, los proyectos de análisis de pastillas suelen recurrir a los medios de comunicación. La información distribuida por lo medios colabora para diluir los mitos emparentados con los peligros y efectos de las sustancias psicoactivas y propiciar debates sobre sustancias ilícitas de un modo más racional que antes.

Se cree que las campañas de prevención sobre el contenido peligroso e inesperado de las pastillas de éxtasis podrían incentivar una conducta más cuidadosa para con las drogas sintéticas en general y, en consecuencia, disminuir la cantidad de consumidores de sustancias ilícitas. Sin embargo, aún no se ha logrado confirmar esta hipótesis.

\section{B. LAS TÉCNICAS DE ANÁLISIS: HETERO- GÉNEAS}

Al hablar de heterogeneidad nos referimos en primer lugar a las variadas técnicas de análisis que se emplean. Éstas se distinguen por la exactitud de la información cualitativa (qué substancias hay exactamente en las pastillas) y cuantitativa (en qué concentración), así como también por los costos:

\footnotetext{
(7) Esto incluye temas tales como patrones de consumo dentro de una sub-cultura específica, estimación subjetiva de riesgos, métodos de reducción de daños particulares y evaluaciones subjetivas de proyectos de prevención, consumo de polidrogas, surgimiento de nuevas sustancias en el Mercado, posibles abusos y adicciones, potenciales daños a largo y a corto plazo, entre otros.

(8) Para obtener información más específica sobre los potenciales beneficios de los proyectos de análisis de pastillas, ver: Schroers, Artur (1999), Die Zukunft hat schon begonnen? Perspektiven der Sekundärprävention o Gesundheitsförderung im Bereich „neuer Drogen“ mit Hilfe von Drug-checking und Monitoring. In: Kammer (ed.): Jugend Sucht Hilfe. Sekundärprävention in der Jugendhilfe. Nürnberg: emwe-Verlag. (9) Cousto, Hans (1997), Drug-checking. Qualitative und quantitative Kontrolle von Ecstasy und anderen Substanzen. Solothurn: Nachtschatten, trata más específicamente el tema de la credibilidad de las personas que imparten infomación sobre los efectos y peligros de las sustancias psicoactivas.
} 
Los kits de análisis rápidos brindan resultados inmediatos sin gastos significativos, pero la información específica no alcanza para determinar exactamente qué derivados anfetamínicos hay en la pastilla ni brinda detalles sobre la dosis. El Marquis test, por ejemplo, es una prueba de reacción de color que detecta la presencia de sustancias de la familia del éxtasis (MDMA, MDA, MDE) pero no logra identificarlas o indicar si hay otras sustancias presentes. Sin embargo, existen algunos químicos que estos análisis no logran detectar, por lo que el hecho de que el resultado dé positivo ante una sustancia semejante al éxtasis, no significa que la pastilla sea pura o segura. Podrán contener una amplia gama de químicos, sanos o peligrosos, tales como PMA que no evidencia ningún cambio de color. Ante este tipo de casos no esclarecidos, algunos Estados Miembro optan por enviar las muestras a sus laboratorios oficiales para completar la información.

Por un lado, hay proyectos que se basan únicamente en la información sobre el aspecto (forma, logo, tamaño). En este caso, se confronta la pastilla con las listas de pastiIlas analizadas anteriormente por otros proyectos, sin que se haga un análisis propiamente dicho.

Los tests inmunológicos se basan en la reacción de un anticuerpo específico frente a determinada sustancia (droga) y, de alguna manera, esta reacción se hace evidente. En la mayoría de los casos, se usa los tests inmunológicos de drogas de orina que hay en el mercado.

La cromatografía es la separación de una sustancia en sus diferentes componentes. Se lo usa mayormente para identificar y determinar los componentes químicos de una sustancia compleja.

Los proyectos que usan tecnología muy avanzada (por ejemplo, HPLC - High performance liquid chromatography) logran conocer en poco tiempo (5-15 minutos) todas las posibles sustancias que pudiera contener la pastiIla, en términos cualitativos y cuantitativos; así se sabe también las dosis que la componen. Es obvio que esta tecnología implica gastos, logística (transporte) y una pericia profesional elevada. Sin embargo, entre este método y los kits rápidos de reacción de color, hay una gran gama de tecnologías (tales como Thin Layer Chromatography (TLC), Gas Chromatography (GC) o pruebas inmunológicas) menos costosas, menos exactas, menos móviles y que llevan más tiempo que la HPLC.

Es importante señalar que se supone que la credibilidad ante el grupo diana aumenta esencialmente con la calidad y exactitud de la información farmacológica y contextual. Para poder llevar a cabo su tarea de prevenir e informar, los proyectos de análisis de pastillas deben garantizar una información rápida y confiable, desde el punto de vista de la cantidad y calidad. La identificación de las pastillas es rápida, pero no resulta nada confiable. Los Marquis Tests son rápidos y baratos, pero su efectividad se reduce sólo a un grupo pequeño de sustancias psicoactivas. En cuanto a la calidad de los resultados, debemos reconocer que no ofrecen información acerca de las posibles adulteraciones o sustancias inesperadas, tales como PMA. Como así tampoco ofrecen información sobre las cantidades. Los tests de reacción de color deberían utilizarse sólo en combinación con las listas de identificación de pastillas, tal como se hacía en el proyecto holandés DIMS. No se puede utilizar los tests inmunológicos para hacer identificación de pastillas.

Debido a que el presupuesto para las intervenciones de análisis de pastillas es limitado, el procedimiento más adecuado para estos proyectos sería asociarse a algún equipo que tenga la pericia necesaria en el área de la cromatografía y que cuente con los equipos (algunas técnicas de cromatografía, tal como la cromatografía de líquidos de alta resolución, podría hacerse en combinación con la identificación de pastillas).

\section{ACTIVIDADES COMPLEMENTARIAS}

La mayoría de los proyectos llevan a cabo otras actividades junto con el análisis de pas- 


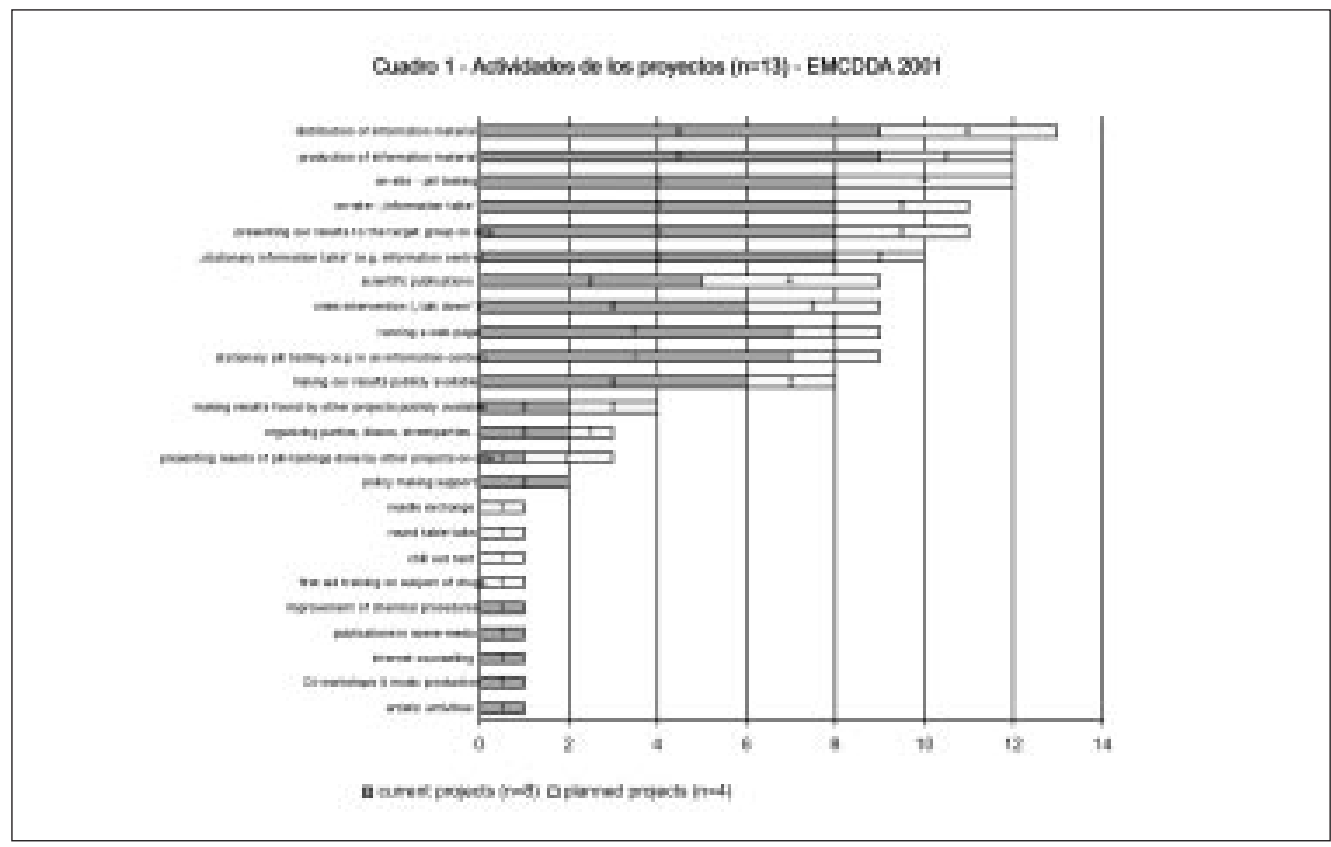

tillas. En general, ofrecen charlas informativas y ayuda en situaciones críticas. Entre las actividades adicionales que se llevan a cabo está la publicación de artículos científicos o la publicación de una página web, con el objetivo de brindar una información objetiva a los grupos diana.

Especialmente los proyectos de Francia (Médecins du Monde http://www.medecinsdumonde.org/2missions/france/RDR/nos $\% 20$ actions3.htm) y Bélgica utilizan estas intervenciones para obtener más información sobre las culturas de consumo y contactar a los consumidores para charlas. En realidad, uno de los elementos más importantes para las intervenciones de análisis de pastillas, y también para los mismos proyectos, consiste en hablar con los visitantes acerca de los efectos y riesgos de las sustancias psicoactivas y del proyecto en sí.

Temas principales de las charlas informativas (OEDT, 2001)

- El proyecto en sí

- Efectos y riesgos de las sustancias psicoactivas

- Composición de las pastillas
- Riesgos relacionados con la combinación de las diferentes sustancias

- Reducción de riesgos y consumo seguro

- Problemas físicos y psicológicos

- Problemas sociales (por ejemplo, en la familia o en la escuela)

- Política de drogas y marco legal

- Set y setting del consumo de drogas

- Cómo apoyar a un amigo que abusa de las drogas

\section{Temas principales de las charlas informati-} vas (OEDT, 2001)

- El proyecto en sí

- Efectos y riesgos de las sustancias psicoactivas

- Composición de las pastillas

- Riesgos relacionados con la combinación de las diferentes sustancias

- Reducción de riesgos y consumo seguro

- Problemas físicos y psicológicos

- Problemas sociales (por ejemplo, en la familia o en la escuela)

- Política de drogas y marco legal

- Set y setting del consumo de drogas

- Cómo apoyar a un amigo que abusa de las drogas 
Sin embargo, en este contexto, "información" no significa seguir el paradigma de prevención y tratar de informar que cada droga ilegal es peligrosa de por sí y en todos los casos. Este tipo de información no resulta creíble para los habitués de fiestas raves o eventos similares. Generalmente, este tipo de personas no se reconoce como "consumidores" y mucho menos como "adictos", por lo que no es frecuente encontrarlos en los centros de asistencia psicológica o de tratamiento habituales. Por todo esto, el punto principal aquí es proporcionar información objetiva del tema sin tomar una actitud moralista. Si bien a la mayoría de las personas le interesa su salud, este hecho no los inhibe de consumir drogas.

\section{PROBLEMAS Y CONSIDERACIONES LEGALES}

Para poder analizar una pastilla de éxtasis o de cualquier otra sustancia, es menester que la misma sea suministrada por los potenciales consumidores. Los técnicos de los proyectos necesitan manipular la pastilla para poder analizarla, si a esto se le suma el hecho de que los análisis de pastillas constituyen un fenómeno relativamente nuevo, es lógico que aún haya bastante incertidumbre en cuanto a su legislación, pues el análisis puede implicar que un técnico reciba, toque, manipule y devuelva substancias ilegales. Para evitar problemas formales con la legislación austriaca, los técnicos de ChEckiT! por ejemplo piden a los potenciales consumidores que ellos mismos raspen una porción de su pastilla dentro de los instrumentos de análisis. Así evitan de tocar las substancias que están bajo control. La policía no se aproxima a los stands donde se efectúa el análisis.

En general, para llevar a cabo un proyecto de análisis de pastillas, se necesita de un mínimo de apoyo político y buena predisposición por parte de la policía local. Debe hacerse un intercambio de opiniones o acuerdos con la policía para evitar que actúen en los

\section{Situación legal (EMCDDA 2001)}

El primer proyecto Europeo y con más acreditación oficial de todos es el DIMS (=Drugs Information Monitoring System, en http:// www.trimbos.nl/trimbos/DIMS.html) de Holanda. Holanda es el único país en el que el análisis de pastillas forma parte de la política oficial de drogas desde 1990. En todos los demás países, los responsables deben recurrir a normas regionales, opiniones legales ad hoc, o acuerdos especiales. En Alemania, por ejemplo, un proyecto piloto en Berlín fue bloqueado por restricciones legales, mientras que otros en Hannover y Münster trabajan sin mayores obstáculos (Techno-Netzwerk-Berlin, 1999). La base de los reglamentos en Austria, Bélgica y Holanda es predominantemente el objetivo científico. ChEckiT! en Viena cuenta con el apoyo de varios actores sociales debido a su combinación de investigación y prevención. A pesar de que para la ley francesa el análisis de pastillas en general es ilegal, el gobierno federal subsidia algunos proyectos de análisis de pastillas, aunque a los potenciales consumidores no se les suministra ningún tipo de información sobre su composición, a menos que contengan sustancias muy peligrosas.

lugares en los que se realizan los análisis de pastillas in-situ, especialmente si la policía, tal como ocurre en la mayoría de los países europeos, está obligada por ley a intervenir en casos potenciales de drogas ilícitas. Es muy frecuente que exista este tipo de cooperación, o al menos que se produzca un intercambio de información con la policía. En general, los proyectos se muestran satisfechos con estos contactos. ChEckiT! ha informado que la policía respalda sus objetivos y no interviene. Es más, existen algunas organizaciones que regularmente mantienen intercambio de información sobre sustancias y tendencias con la policía. Igualmente, es importante aclarar que los proyectos no suministran ningún tipo de información acerca de sus clientes. 


\section{E. El problema de la evaluación}

De los ocho proyectos incluidos en el estudio del OEDT, sólo cinco han analizado aunque sea algunas de las cuestiones de evaluación, y sólo dos expresan haber analizado "la mayoría de las cuestiones" o haber hecho una evaluación completa tal como se lo aconseja en la guía de evaluación del OEDT ${ }^{10}$. La evaluación de sus procesos y objetivos es un paso necesario en cualquier proyecto profesional y un requisito básico para conseguir fondos públicos. Sin embargo, hay algunos motivos por los que hasta este momento no existe una cultura de evaluación en el ámbito del análisis de pastillas. El análisis de pastillas constituye un concepto relativamente nuevo en el área de salud pública y prevención, por lo que aún no existe ninguna información o experiencia que se base en los instrumentos de evaluación. Por otra parte, hay muchas organizaciones en el área de análisis de pastillas que no cuentan con los recursos económicos ni la pericia necesaria para diseñar los elementos de evaluación adecuados para los proyectos de análisis de pastillas. Es importante señalar que el proceso de evaluación se simplifica a medida que se cuenta con más conocimiento y se sabe lo que ocurre alrededor.

A modo de ejemplo, podría verse el caso del proyecto ChEckiT!, de Austria, que utiliza a diario los siguientes indicadores:

\begin{tabular}{|c|c|c|}
\hline $\begin{array}{l}\text { Evaluación de } \\
\text { proceso de ... }\end{array}$ & Variables e Indicadores & Métodos \\
\hline \multirow{6}{*}{ Análisis in-situ } & Cantidad de jóvenes contactados & \multirow{6}{*}{$\begin{array}{l}\text { Breve entrevista con la persona } \\
\text { que acerca la pastilla a analizar } \\
\text { (cuestionario) }\end{array}$} \\
\hline & Sexo y Edad & \\
\hline & Cantidad de pastillas analizadas & \\
\hline & $\begin{array}{l}\text { Diferentes variables relacionadas } \\
\text { con la descripción de la pastilla }\end{array}$ & \\
\hline & $\begin{array}{l}\text { Información sobre si el cliente consume la } \\
\text { pastilla antes de conocer los resultados del análisis }\end{array}$ & \\
\hline & $\begin{array}{l}\text { Preguntas acerca de motivos que lo llevaron a hacer } \\
\text { el análisis, consumo y comportamientos de riesgo }\end{array}$ & \\
\hline \multirow{4}{*}{$\begin{array}{l}\text { "Charlas } \\
\text { informativas" } \\
\text { in-situ }\end{array}$} & Cantidad de personas contactadas & \multirow{4}{*}{$\begin{array}{l}\text { Planilla rápida (una hoja que los } \\
\text { miembros del personal puedan } \\
\text { llevar con ellos para registrar } \\
\text { fácilmente contactos y los } \\
\text { temas de las charlas) }\end{array}$} \\
\hline & Sexo y Edad & \\
\hline & Temas abarcados en las charlas informativas & \\
\hline & $\begin{array}{l}\text { Problemas que se presentan y sugerencias para } \\
\text { mejoras }\end{array}$ & \\
\hline Página web & Cantidad de visitas & \\
\hline & $\begin{array}{l}\text { Cantidad de visitantes que participan de las } \\
\text { salas de chat }\end{array}$ & \multirow{3}{*}{ Contadores de visitas } \\
\hline & $\begin{array}{l}\text { Cantidad de preguntas personales transmitidas } \\
\text { por la red }\end{array}$ & \\
\hline & Valoración de la "calidad" de las preguntas personales & \\
\hline
\end{tabular}

Los equipos móviles usan el tiempo que los clientes esperan delante del stand de análisis para pasar cuestionarios o hacer entrevistas (por ejemplo, preguntas sobre los motivos de las pruebas, consumo de drogas y comportamiento de riesgo).

Sin embargo, cuando se toma en cuenta las circunstancias en las que se trabaja en las

(10) http://www.emcdda.org/infopoint/publications/manuals.shtml 
fiestas raves, se verá que sólo es posible llevar registro de un modo rápido y superficial. Se hace muy difícil, y hasta casi imposible, hacer una evaluación de los resultados en este contexto específico. En la mayoría de estos proyectos, se contacta a los visitantes sólo una o dos veces, y es muy difícil saber si la información suministrada eventualmente lleva a un consumo más seguro en comparación con los consumidores que no han recibido esta información. Este hecho dificulta enormemente cualquier proyecto de evaluación de resultados estructurado.

En resumen, hasta el momento, no se conoce el "estado real" de la evaluación de los proyectos de análisis de pastillas $y$, teniendo en cuenta las circunstancias, se hace imposible suministrar esta información a los políticos para que puedan decidir con fundamento científico sobre la importancia de contar con intervenciones de análisis de pastillas in-situ.

Afortunadamente, en el año 2002, la Comisión Europea financió un estudio de evaluación en tres ciudades (Hannover, Ámsterdam y Viena) con 750 personas en total, que ha analizado las siguientes hipótesis:

(1) el análisis de pastillas posibilita el contacto con consumidores que de otro modo no serían alcanzables

(2) los mensajes de alerta son recibidos con más credibilidad en el contexto del análisis de pastillas

(3) los consumidores están mejor informados

(4) Ios análisis de pastillas facilitan el monitoreo y la observación del mercado.

Las tres primeras hipótesis fueron confirmadas; la cuarta, sólo en ciertas circunstancias.

A su vez, también se confirmó la hipótesis sobre prevención primaria: el análisis de pastillas no estimula el consumo de drogas sintéticas, incita a los potenciales consumidores a no iniciar el consumo y desmitifica las drogas sintéticas entre consumidores (Benschop, 2002, o en versión abreviada en http://eddra.emcdda.eu.int:8008/eddra/plsql/s howQuest?Prog_ID=2828).

\section{F. LOS DEBATES SOBRE EL ANÁLISIS DE PASTILLAS}

Desde que el OEDT publicó en 2001 el estudio exploratorio a escala europea en que se basa parte de este capítulo (en inglés en http://www.emcdda.org/multimedia/project_r eports/responses/on-site_pill_testing_ sum.pdf (resumen) y en http://www.emcdda.org/multimedia/project_reports/responses/pill_testing_report.pdf (informe completo)), este informe provocó debates muy encendidos (www.lalibre.be/article_print. phtml?art_id=72058) entre los políticos de algunos países y algunos acusaron incluso al OEDT de promover estas intervenciones de análisis de pastillas y de dañar así a los jóvenes (Corriere della Sera, 2001). Todo esto se debió sólo a la publicación de un simple informe científico y descriptivo que ofrece únicamente una visión global, sistemática y comparativa de los proyectos de análisis de pastillas y que no contiene argumentaciones ni a favor ni en contra sobre este tipo de intervenciones. A su vez, dentro de la literatura científica también existen argumentaciones que cuestionan el valor de estas intervenciones (Winstock 2001) y que serán discutidas en este capítulo.

Por todo esto conviene revisar los distintos aspectos y argumentos en pro y en contra del análisis de pastillas, puesto que el informe descriptivo mencionado carecía de este análisis.

\section{a) "El problema de la adulteración de pas- tillas es cada vez menor"}

En Holanda y Francia se ha detectado últimamente que la pureza de las pastillas de éxtasis aumenta y que la probabilidad de que contengan otras sustancias es cada vez menor (Delile, J-M., Gachie, J-P., 2002). Este argumento le quitaría fundamento a los proyectos de análisis de pastillas, dado que el riesgo de consumir sustancias tóxicas inesperadas parece en disminución. El problema es que este fenómeno sólo se observa en el oeste Europeo. Por su parte, los proyectos alemanes de análisis in situ relatan que las 
listas de pastillas de origen holandés que se utilizaban hasta el momento son cada vez menos útiles a la hora de identificar las pastillas, porque aparentemente aumenta la contaminación del mercado con pastillas provenientes del este europeo, donde el grado de pureza es más oscilante. Otro aspecto importante es que el proyecto ChEckiT! de Viena demuestra que los casos de pastillas con dosis excesivas de éxtasis (>120 mg) vienen en aumento, lo que constituye también un riesgo considerable, a pesar de la pureza del contenido.

Si la adulteración de las pastillas vendidas como éxtasis ya no se considerara un problema relevante, la Unión Europea no mantendría el sistema de alerta rápida que intenta justamente coordinar los sistemas de información sobre nuevas sustancias dentro de las pastillas, la evaluación de riesgos y la introducción de medidas legislativas ${ }^{11}$. De este modo, los mismos responsables políticos confirman que todavía hay un peligro serio que justifica la permanencia del control sobre la adulteración de las pastillas. Al mismo tiempo, se defiende también la voluntad de los responsables de los proyectos de análisis de pastillas de transmitir la información sobre posibles sustancias contaminadoras a su grupo diana. Si bien es cierto que hay algunos proyectos en este ámbito que se basan en la presunción de que el único problema de las pastillas es la adulteración y lo utilizan como estandarte para su legalización ("si las pastillas fueran legales no habría adulteración y los análisis no serían necesarios"), se debe tomar en cuenta que ésta es la consecuencia lógica de un discurso público y oficial que resalta el riesgo de la adulteración, quedando así relegados los riesgos de consumo en sí aún inciertos ante la percepción pública. En otras palabras: la gran preocupación que existe en el ámbito político por la posible contaminación de las pastillas envía una señal clara a los profesionales y al público de que el problema de las pastillas reside en la adulteración y no en el consumo en sí (de las pastillas). En esta discusión, los proyectos de análisis de pastillas sólo responden a una imagen dentro del discurso público (la imagen de que el peligro es la adulteración y no las pastillas en sí) aunque no son responsables de la creación de esta imagen. En este texto, se parte de la aceptación de esta situación y se discute solamente las diferentes estrategias para promover un uso menos peligroso, como prevención secundaria. Por este motivo, aquí no se cuestiona el valor de la prevención primaria en el ámbito recreativo ni la validez de los mensajes de no-consumo.

\section{b) "El análisis de pastillas sugiere una pseudo-seguridad traicionera"}

Éste es el argumento principal del artículo de Adam Winstock (Winstock, 2001). Los proyectos que sólo usan kits de análisis rápidos (Marquis) no logran detectar con certeza si una pastilla realmente contiene éxtasis, puesto que apenas llegan a identificar el grupo químico al que pertenecen. Por otro lado, tampoco tienen la capacidad de excluir la posibilidad de que una pastilla contenga otras

(11) Alertados por las incidencias esporádicas de nuevas sustancias o de sustancias no esperadas dentro de las supuestas pastillas de éxtasis, los países de la Unión Europea establecieron un Sistema de Alerta Rápida sobre Nuevas Drogas Sintéticas, coordinado por el OEDT en colaboración con EUROPOL. Cuando en decomisos de drogas aparecen nuevas sustancias dentro de las pastillas ofrecidas en el mercado de un país, se alerta sobre dicha sustancia, iniciándose así un proceso de evaluación común de los riesgos de la sustancia en cooperación con todos los Estados miembros y la Comisión Europea y se recopila toda la información de la que disponga cada uno de los Estados miembros. El resultado del ejercicio es un informe de la evaluación de los riesgos de estas sustancias. Hasta este momento, el OEDT ha publicado informes sobre MBDB, 4-MTA, Ketamina y GHB. De a cuerdo con los resultados de los informes, se decide si se da o no el aviso y consejo a los estados miembros para controlar o prohibir las sustancias en cuestión, y para la difusión de la información pertinente. Esto puede tener impacto en los contratos internacionales de control de precursores de dichas sustancias. El sistema de alerta rápida hace un uso parcial del DIMS y de sistemas parecidos para la recogida y re-difusión de la información a los grupos-diana. Como en todos los sistemas preponderantemente políticos surge la pregunta de cómo puede transformarse y usarse esta información producida en mensajes contiguos para la prevención o para estrategias de reducción de daños. Posiblemente las intervenciones de "pill testing" podrían servir de eslabón entre estas dos áreas. Las publicaciones provenientes del sistema de alerta rápida se pueden pedir en http://www.emcdda.org/publications/publications_adhoc.shtml, y el informe sobre MBDB se puede bajar directamente de http://www.emcdda.org/multimedia/Publications/MBDB.pdf 
sustancias dañinas ni alertar ante concentraciones altas de MDMA (éxtasis) y, de este modo, se abre la posibilidad de que estos proyectos den una imagen pseudo-científica ante sus clientes, dejándolos con una sensación errónea de haber excluido posibles peligros. Si bien el argumento es válido, parte de premisas parcialmente equívocas, ya que los grandes e importantes proyectos de análisis de pastillas (en Francia, Holanda ${ }^{12}$, Austria) usan tecnologías avanzadas, tal como el autor exige, que permiten identificar dosis y contenido, y excluir la presencia de contaminaciones. Sin embargo, es verdad que existen algunos pequeños proyectos que se basan en kits de análisis, pero esto se debe en gran parte a los problemas legales que dificultan la posibilidad de contar con el apoyo y la cooperación de instituciones con la pericia y tecnología necesarias (por ejemplo, institutos farmacológicos). En el caso de Alemania, por ejemplo, se prohibió que ciertos proyectos establezcan acuerdos con institutos farmacológicos.

Si bien los kits de análisis rápidos no logran identificar fehacientemente los contenidos de las pastillas, tienen no obstante un efecto cognitivo importante sobre los consumidores: las investigaciones cualitativas llevadas a cabo en Irlanda del Norte confirman que ellos, como tienden a obtener información sobre sus drogas de fuentes personales o de amigos, tienen preferencia por un mismo "logo" en las pastillas, ya probado o recomendado por otros. Se fían de los "logos" como si éstos representaran una garantía de contenido y dosis estables, tal como ocurre con los medicamentos (McElrath \& McEvoy, 1999). Sin embargo, un simple análisis de las pastillas podría desmitificar esta creencia en "marcas registradas" al demostrar (aunque sea sin demasiada calidad analítica) que un logo no garantiza una correspondencia con el contenido esperado.

Un punto muy crítico en la argumentación sobre la pseudo-seguridad es que la comple- jidad de las intervenciones de análisis de pastillas en lugares de ocio se ve reducida a meras cuestiones de metodología farmacológica, omitiendo algunos aspectos preventivos mucho más importantes, que veremos más adelante.

\section{c) "Analizar pastillas en lugares de ocio implica aceptar e incluso promover el con- sumo. Así enviamos el mensaje equivoca- do a los jóvenes"}

Éste es el argumento más frecuente y común. Se alega que los análisis de pastillas, hechos visibles y abiertos en discotecas y clubes, envían mensajes de aceptación del consumo a los jóvenes que todavía no han considerado el uso de drogas. Esto es parte del vasto repertorio de argumentaciones contra las intervenciones sociales hacia grupos diana especialmente en riesgo. Lo mismo se dijo hace años sobre los programas de sustitución y de jeringuillas (que simbolizarían la aceptación social del consumo de heroína) y sobre la despenalización del consumo de drogas. No hemos recibido ninguna información que afirme que estas medidas hayan facilitado la aceptación del consumo de heroína en el medio social. En principio, resulta problemático que las intervenciones especializadas, pragmáticamente dirigidas a grupos de la población bastante restringidos, sean continuamente objeto de análisis filosóficos que los extrapolan y alargan teóricamente hacia un significado social y global. De hecho, se puede suponer que las actividades de prevención específicas sean socialmente percibidas como destinadas específicamente a un pequeño grupo diana en riesgo y no como un mensaje o significado para toda una sociedad. En las regiones europeas en donde los conceptos pragmáticos prevalecen por sobre los filosóficos en la salud pública, se observa bastante cooperación por parte de la policía, de las autoridades locales y, obviamente, de los organizadores en pos de una búsqueda

(12) En el ámbito de DIMS, los tests ahora ya no se efectúan in situ, sino fuera del contextos de las fiestas, pero también se incluye contacto personalizado. 
común de soluciones prácticas y adecuadas para los problemas derivados del consumo de drogas. En estos casos, la policía sigue sus propios objetivos de prevención y no interviene. La mera existencia de estos proyectos ya reduce los problemas y, en consecuencia, la necesidad de intervención en las fiestas. Es evidente que es más sencillo desarrollar estrategias pragmáticas en cooperación con las autoridades locales y servicios de salud si los proyectos se abstienen de adornar su práctica profesional con largas construcciones ideológicas en torno al análisis de pastillas y no intentan introducir discusiones fundamentalistas sobre el concepto de reducción de daños o sus implicaciones político-filosóficas.

La evaluación de los proyectos de análisis de pastillas (Benschop 2002) no encontró ningún efecto de instigación al consumo entre los no-consumidores, pero logró confirmar que muchos de los consumidores que entraron en contacto con esos proyectos no habían tenido contactos previos con servicios preventivos o de salud. Merece la pena recordar que la característica más sobresaliente del análisis de pastillas es justamente el hecho de que atrae SOLA Y ESPECIFICAMENTE al grupo diana que pretende contactar: aquellos que acaban de comprar pastillas de éxtasis. Lógicamente, son sólo ellos quienes se aproximan a las zonas más calmas (Van der Wijngaart, 1998) de las fiestas o discotecas y es allí donde se ubican las instalaciones de estos proyectos. Esto quiere decir que no hay una interacción directa con aquella mayoría de gente que acude a las fiestas y se divierte sin consumir drogas ilícitas. Sabemos que este tipo de consumidores no se considera consumidores típicos de drogas, "junkies". Tienen la auto-imagen de estar socialmente integrados y de usar drogas de una manera controlada y segura (Griffiths et al., 1997). Este grupo no se acerca fácilmente a los servicios tradicionales de ayuda o prevención porque no consideran que tienen un "problema con las drogas". La posibilidad de analizar las pastillas y tener una charla informativa individual sobre riesgos y estilos de vida, suele atraer a este grupo diana que, en general, es difícil de contactar.

Se podría decir que éste es un problema que radica en las medidas regulares, es decir, las estrategias informativas en las que no hay análisis de pastillas, puesto que no logran controlar los distintos mensajes que se envían a los distintos grupos diana. Al distribuir postales, boletines y panfletos sobre el uso menos arriesgado de drogas en discotecas, no se sabe a ciencia cierta si el destinatario contactado realmente consume drogas o si ni siquiera había contemplado la idea hasta ese momento. El mismo principio se aplica para las campañas de información preventiva en medios de comunicación, tal es el caso de las revistas para jóvenes donde se transmite y discute información muy específica sobre sustancias a todos los públicos, inclusive a aquellos que ni piensan en consumir, como si el consumo de esas substancias fuera corriente y normal. Este dilema de enviar "señales" a destinatarios equivocados es menor en el caso de los proyectos de análisis de pastillas puesto que atraen sólo a los destinatarios deseados. Por eso el análisis de pastillas no envía "mensajes peligrosos" a la juventud. Las medidas de información regulares corren incluso más este riesgo, porque son menos específicas y pueden influenciar la percepción de normas (Hansen, 1992).

\section{d) "Analizar pastillas y devolverlas con información sobre el contenido da la idea de que las drogas sintéticas son inocuas"}

Este argumento se basa en la suposición de que el personal de proyectos de análisis de pastillas devuelva las pastillas que realmente contienen éxtasis como si fuera un certificado de seguridad, asegurando que se pueden consumir sin problemas porque contienen lo esperado. En realidad, la estrategia comunicativa de estos proyectos es hacer uso del momento en que se comunica los resultados del análisis para reflexionar principalmente sobre el uso de pastillas y los riesgos individuales que hay que tener en cuenta. Por ejemplo, es muy importante que los 
potenciales consumidores sepan - incluso si la pastilla analizada contiene de hecho solo MDMA - que la probabilidad de alteraciones neurofisiológicas aumenta proporcionalmente con la cantidad de MDMA consumida y que puede haber daños a largo plazo. Esto es el tipo de mensaje que se pasa en el caso del ChEckiT! y de varios otros programas (EMCDDA 2001). Sin embargo, cabe mencionar que en otros proyectos, en general, la interacción se redujo a informar sobre el contenido de la pastilla sin que hubiera charlas preventivas o de cualquier otro tipo (Van der Wijngaart, 1998).

Por otra parte, las encuestas escolares de ESPAD llevadas a cabo en varios países (Hibbell et al., 2000) sugieren que en la representación social que existe sobre las drogas YA HAY una imagen de que las drogas sintéticas no son demasiado peligrosas: en todos los países de la UE, con excepción del Reino Unido e Irlanda, los estudiantes consideran que un paquete o más de tabaco por día es más peligroso que consumir éxtasis una o dos veces (tómese en cuenta que España no participa del ESPAD y que los datos de las encuestas escolares, según el Observatorio Español sobre Drogas, no confirman esta observación para su país). Es posible que su aparente similitud con los medicamentos sugiera una cierta calidad farmacéutica (en dosis y contenido). Las charlas informativas que acompañan los análisis brindan la posibilidad de dilucidar estos mitos sobre la seguridad farmacéutica de las drogas sintéticas e informar cuidadosa y objetivamente sobre los riesgos reales. La información realista sobre riesgos frecuentes no promueve el abuso de drogas.

\section{e) "Estos proyectos pueden ser usados por los traficantes, incluso con fines publi- citarios"}

No se puede descartar del todo la idea de que los traficantes de drogas sintéticas podrían abusar de estos servicios para analizar la calidad de su propia mercancía. Hasta ahora, no hubo evidencia que lo confirme. Sin embargo, es tan difícil confirmar esta hipótesis como refutarla. La mayoría de los proyectos de análisis de pastillas ha contemplado maneras para evitarlo, por ejemplo, no se acepta que una misma persona traiga varias pastillas para analizar. El hecho de que el análisis esté estrechamente ligado a charlas de información puede también disminuir este tipo de abuso. Tal como se lo explica en uno de los proyectos: "No somos un servicio público de traficantes; la gente deberá acercarse a nosotros para hablar sobre su consumo".

\section{f) "La información puede provocar reaccio- nes paradójicas"}

Esto hace referencia al hecho de que se ha observado que los consumidores, al enterarse de que su pastilla contenía menos éxtasis del esperado, tomaban una dosis mayor (Winstock, 2000). Éste es un argumento importante que no se puede ignorar. Por otro lado, hay que admitir este mismo efecto se da como consecuencia de cualquier medida de información: si los consumidores se informan a través de la prensa juvenil o de boletines de que las pastillas disponibles en el mercado negro no contienen cantidades iguales de MDMA, tenderán siempre a tomar una pastilla más en el caso de no tener el efecto esperado. Éste es el peligro particular de las drogas sintéticas fuertes con efecto retardado, como por ejemplo la 4-MTA. Los proyectos de análisis ofrecen, al menos, la posibilidad de controlar individualmente el modo de presentar la información sobre la dosis de MDMA en las pastillas y de controlar el modo en que el cliente la recibe y entiende (Delile, J-M., Gachie, J-P., 2002), a partir de sus reacciones y preguntas. Los consumidores encuestados por Van der Wijngaart et al. (1998) indicaron que la oferta del análisis de pastillas no los llevó a un aumento de consumo en general. La reciente evaluación de los proyectos de análisis de pastillas llegó a la misma conclusión (Benschop, 2002). 


\section{g) "Hay alternativas mejores"}

Hay voces que defienden que para informar a los jóvenes acerca de los riesgos asociados al consumo de drogas sintéticas sería más adecuado utilizar la prensa juvenil, ya que así se evita dar señales de aceptación pública del consumo o posesión de pastillas (véase arriba). En la prevención es importante fraccionar el contenido de mensajes según los grupos (y sub-grupos) diana: un adolescente joven y abstemio no debería recibir los mismos mensajes o consejos que se le daría a un adulto joven que ya ha experimentado esporádicamente algunas drogas ligeras. Sin embargo, posiblemente ambos lean las mismas revistas para adolescentes y frecuenten los mismos lugares de ocio. Cuando las estrategias de prevención se basan demasiado en formas unidireccionales de información, como es el caso de revistas y campañas mediáticas, se corre el riesgo de no alcanzar con suficiente especificidad a los respectivos grupos diana. Dar consejos sobre uso de drogas menos peligrosas a jóvenes abstemios es tan peligroso como teñir de un dramatismo exagerado los peligros a corto y largo plazo de las drogas sintéticas para consumidores que ya tienen experiencias. Los proyectos de análisis de pastillas son suficientemente específicos y selectivos como para evitar este problema ya que atraen selectivamente a los consumidores, o potenciales consumidores, y sólo a ellos les brinda la información específica sobre disminución de riesgos o los invita a reflexionar sobre hábitos y causas de consumo. El estudio de Benschop logró confirmar esta hipótesis (Benschop 2002). De este modo, se cubre la brecha que se evidenció en un estudio hecho por el OEDT sobre intervenciones preventivas en el ámbito recreativo (Tossmann et al., 1999): la relativa falta de intervenciones destinadas a consumidores problemáticos.

Por otro lado, comparte con las estrategias ya corrientes de grupos de pares (peer group approaches) la interactividad de la información, un imprescindible factor de éxito ya conocido por las intervenciones preventivas (NIDA 1997, Sindballe 2000). A diferencia de los boletines, videos y revistas, la información no es unidireccional, puesto que se puede responder a reacciones, preguntas $y$ situaciones individuales y no se reduce sólo a la información sobre sustancias. En este capítulo (p. 12) se presentó una lista con los distintos temas de estas charlas informativas. Se observará que van mucho más allá de la mera información sobre sustancias e incluyen el contexto individual y social de consumo, los motivos de uso, la situación privada, etc. La información individualizada resulta también más realista y factual al ser transmitida por profesionales que utilizan un lenguaje adaptado a los destinatarios. En prevención, es importante que los elementos de información sean realistas, correctos, concretos y equilibrados, sin que se haga exageraciones o que se ponga excesivo énfasis en los posibles peligros (NIDA, 1997). Esto es un principio universal en prevención y en la mayoría de los estados miembros hace ya tiempo que se ha aprendido la lección: hay que ser cauteloso con el tono de los mensajes preventivos. Los jóvenes destinatarios disponen siempre de fuentes de información informal (amigos, Internet) y algunas veces tienen sus propias experiencias con drogas. Si la información proveniente de fuentes oficiales de prevención evoca riesgos y peligros que contradicen sus experiencias individuales o colectivas, es probable que a partir de entonces los jóvenes rechacen sistemáticamente toda información preventiva oficial (Uhl 2000). El problema de la credibilidad es menor en las intervenciones directas, interactivas y específicas, como es el caso de los equipos móviles para el análisis de pastillas. Es verdad que dentro de la prevención secundaria el papel de la información se basa más en presunciones que en evidencias científicamente comprobadas. Por ende, al igual que tantos otros colegas, se supone que para esta discusión es correcto el paradigma que dice que "la información sobre riesgos y sobre estrategias de diminuirlos es preventiva", sin poder aportar las fuentes de evidencia necesarias. En la mayoría de los estados miembros se observa que las estrategias de información están teniendo una renaissance en prevención secundaria o 
Cuadro 2. Profesionales trabajando en proyectos de análisis de pastillas. EMCDDA 2001

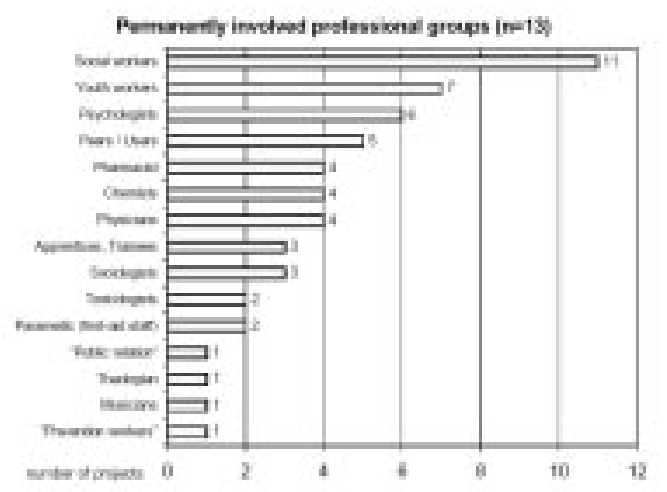

reducción de riesgos en conceptos como informed choices (tomar decisiones a partir de una buena información) y risk-competence (capacidad para controlar riesgos). Implícitamente, estos conceptos y paradigmas se aplican en todas las intervenciones en el ámbito recreativo, con o sin análisis de pastillas.

Considero que hay un consenso entre profesionales y expertos en que el elemento más importante en la prevención de daños asociados al consumo de éxtasis no es solamente la información sobre las pastillas, su contenido y efecto, sino sobre las razones (¿búsqueda de sensaciones fuertes? ¿Autotratamiento?), los contextos de consumo (si disponen de suficiente agua y ventilación), el consumo mezclado con otras drogas (legales e ilegales) y una reflexión sobre posibles daños a largo plazo. Para llegar a este nivel de conversación, el personal de los proyectos de análisis de pastillas debe tener buena competencia técnica y comunicativa (véase el cuadro 2), y se debe garantizar que la información transmitida al grupo diana no se reduzca sólo al contenido de las pastillas. Sin embargo, estos aspectos atañen a la formación profesional y al diseño de los proyectos, por lo que son elementos técnicos de implementación que no tienen nada que ver con el contenido.

\section{G. COMENTARIOS FINALES}

Cuando se discute el valor del concepto de análisis de pastillas para estrategias preventivas (o de reducción de riesgos), es legítimo presuponer que cada uno de los proyectos ha de implementarse con calidad y rigor, que el personal tendrá una buena formación (véase cuadro 2, donde se observa que el nivel de formación es bastante alto), que los proyectos realmente se expondrán en zona calmas y retraídas, y que el contacto con los clientes realmente se aprovechará para charlas informativas que no se limiten sólo a la comunicación del contenido de las pastillas. Muchos proyectos cumplen con todos estos requisitos, aunque es posible que haya otros proyectos que sean menos prolijos y cuidadosos en sus procedimientos y menos discretos en su presentación. El hecho de que algunos proyectos de análisis de pastillas no consigan llegar al nivel ideal o que incluso lo usen para promocionar mensajes de legalización de drogas sintéticas, no le quita valor a este concepto, sólo cuestiona su grado y forma de implementación. Análogamente, tampoco se pone en duda el valor del concepto de LA PREVENCIÓN como tal, aunque se sabe que en la mayor parte de la Unión Europa no se implementa de un modo apropiado (véase el Informe Anual del OEDT en www.emcdda.org) o se realizan prácticas insuficientes. 
Al discutir sobre estrategias, debemos partir de la suposición de que se las implementa con calidad, próximo a su concepto ideal, y de que el hecho de que, en la práctica, siempre haya malas implementaciones en este campo, no le quita valor al principio subyacente. En la práctica, sería mucho más recomendable vigilar la puesta en marcha con altos criterios de calidad y apoyar una buena evaluación para monitorear y mejorar estas iniciativas en vez de rechazar tajantemente todo un concepto porque a simple vista parece arriesgado y porque en algunos proyectos hay personas que defienden el derecho a consumir drogas sintéticas.

Este texto no es una defensa al valor científicamente probado del análisis de pastillas. Se trata más bien de una llamada a un debate justo y ponderado sobre la situación, el potencial y los efectos de estos proyectos en la Unión Europea, y contra las especulaciones filosóficas e ideológicas hechas a priori sobre el significado para la sociedad o la juventud. En este momento, se carece de fundamentos científicos suficientes sobre los beneficios o peligros reales de los análisis de pastillas que permitan generalizar en todos los países europeos. Aún no hay evaluaciones rigurosas y la diversidad de formas de puesta en marcha y de actuación, la diferenciación de los mensajes según los diferentes grupos-diana, los distintos intereses de investigación y las consideraciones de coste-beneficio dificultan crear un cuadro concluyente y homogéneo.

Para una prevención holística en ámbitos recreativos y sobre todo para evitar los problemas de salud más sobresalientes en los clubes, hay medidas primordiales e importantes como guías organizativas para clubes y fiestas que en la actualidad se desarrollan en Europa

(véase http://www.clubhealth.org.uk/). Por esto, el análisis de pastillas no es la respuesta más importante a los problemas de salud en discotecas $y$, de hecho, es un tipo de intervención que en la realidad europea no se efectúa en gran escala. Tiene una indicación bien definida y limitada para problemas específicos.
Por lo tanto, no se puede recomendar indiscriminadamente para todos los ámbitos, fines y contextos sociales o culturales en Europa, ni se justifica la actual demonización que se observa en algunas partes: es importante y justo que se pueda discutir e investigar sobre los contenidos y la posible utilidad de estas intervenciones. Según la información de la que disponemos en este momento, parecen indicados para potenciar el impacto de los equipos móviles y contactar a ciertos grupos de riesgo. Las cuestiones metodológicas de carácter puramente farmacológico son, en este contexto, de importancia secundaria.

\section{REFERENCIAS}

Benschop, A. , Rabes, M. , Korf., D.J. (2002) Ecstasy, Prävention und

Pill Testing - Eine wissenschaftliche Evaluationsstudie in drei europäischen Städten - Amsterdam 2002

Corriere della Sera, 2 de Noviembre de 2001: Muccioli: così si danneggiano i ragazzi

Delile, J-M., Gachie, J-P. (2002) Ecstasy et réduction des risques - La place du testing et les analyses de substances. Alcoologie et Addictologie 2002; 24 (4): 311-318.

Forum Européen, Securities drugs-network: The Pertinency of Drug Prevention. Messages Project 1998-1999. Rennes: IMR, 1999

Griffiths, P., Vingoe, L., Jansen, K., Sherval, J., Lewis, R., Hartnoll, R., Nilson, M. (eds.): New trends in synthetic drugs in the European Union: epidemiology and demand reduction responses. EMCDDA Insights Series, $N^{\circ} 1$, 1997.

Hansen, W., School-based substance abuse prevention: a review of the state of the art in curriculum, 1980-1990. 1992; Health Education Research, 7 (3), 403-430.

Hibbell, B., Andersson, B., Ahlström, S., Balakireva, O., Bjarnson, T., Kokkevi, A., Morgan, M., the Swedish Council for information on alcohol and other drugs (CAN), the Pompidou Group of the Council of Europe: Alcohol and drug use among students in 30 European countries. December 2000 
McElrath, K. and McEvoy, K. (1999). Ecstasy use in Northern Ireland. Queen's University, Belfast.

NIDA. Preventing drug use among children and adolescents: a research-based guide. Bethesda, MD, National Institute on Drug Abuse. 1997

Observatorio Español sobre Drogas. Encuestas sobre Drogas a Población Escolar, 1994, 1996, 1998 y 2000. DGPNSD. OFDT - Observatoire français des Drogues et des Toxicomanies: Rapport Sintes n`1 (Jan-Oct 1999).

EMCDDA - European Monitoring Centre for Drugs and Drug Addiction. An inventory of on-site pilltesting interventions in the EU. EMCDDA scientific report. EMCDDA, 2001 (available at http://www.emcdda.org/multimedia/project_re ports/responses/pill_testing_report.pdf)

Sindballe, A-M: Unge og rusmidler - evidensbaseret forebyggelse i skolen. MPH-thesis. Institute of Public Health. Copenhagen University, 2000.

Techno-Netzwerk-Berlin: Drug Checking Konzept für die Bundesrepublik Deutschland, 1999

Tossmann, H.P, Heckmann, W.: Drogenkonsum Jugendlicher in der Techno-Party-Szene. Köln: BzgA, 1997
Tossmann, P., Bold, A., Tensil, M.-T.: Demand Reduction Activities in the Field of Synthetic Drugs in the European Union. EMCDDA 1999, at http://www.emcdda.org/multimedia/project_reports/responses/ddr_nsd_report.pdf

Uhl, A.: "Non-problematic Use" -"problematic use": a paradox. In: Springer, A., Uhl, A. (Eds.) Illicit drugs. Patterns of use - patterns of response Proceeding on 10th ESSD Conference 2000.

Van der Wijngaart, G., Braam, R., de Bruin, D. et al.: Ecstasy and the Dutch Rave Scene. Utrecht University, Addiction Research Institute, 1998

Winstock, A.R., Wolff, K., Ramsey, J., : Ecstasy pill testing: harm minimisation gone too far? Addiction (2001), 96, 1139-1148

\section{PÁGINAS WEB:}

http://www.checkyourdrugs.at/ http://www.energycontrol.org/

http://www.emcdda.org/responses/themes/outreach_pilltesting.shtm 
